Imaging the

vitreous: optical coherence tomography and
ultrasound imaging

M Restori

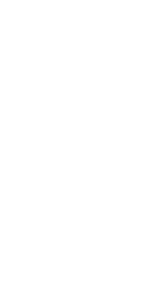

Abstract

Imaging the vitreous in optically transparent and translucent ocular media using OCT and in optically opaque ocular media using ultrasound is discussed.

Optical coherence tomography and colour flow mapping are briefly described. Images of tomograms, B-mode sections, and colour flow maps illustrate the use of these techniques in imaging the vitreous.

Eye (2008) 22, 1251-1256; doi:10.1038/eye.2008.30;

published online 22 February 2008

Keywords: vitreous; fourier domain OCT; B-mode; colour flow mapping

\section{Optical coherence tomography}

'The existence of light allows us to walk along the tightrope between translucency and transparency' — Sir Nicolas Grimshaw.

Optical coherence tomography (OCT) allows cross-sectional imaging of eyes with a micronscale resolution. The requirements are the ocular media should be optically transparent or translucent and the pupil diameter should be a minimum of $4 \mathrm{~mm}$. Patients are seated with the head positioned and stabilised with a chin rest and forehead support. It is common to combine OCT with the fundus image to allow a choice in OCT section locations.

OCT images are comparable to ultrasound B-mode sections, both presenting serial (or radial) cross-sectional images in the same planes and data on the amplitude of echoes scattered from the tissues at measured depths. Hence, terminology is often used interchangeably for both techniques. An important difference between the techniques, however, is how the data regarding echo depth is obtained. The speed of sound in the vitreous is $\sim 1.5 \times 10^{3} \mathrm{~m} / \mathrm{s}$, whereas that of light in the vitreous is a factor of $10^{5}$ times greater at $\sim 2.3 \times 10^{8} \mathrm{~m} / \mathrm{s}$. In ultrasound imaging, separating out the scattered echo data from different depths is by means of measuring time delays of echoes. In OCT, however, the echo data have extremely short time-delays necessitating the use of interferometric techniques to extract the depth information.

\section{Time-domain OCT}

The technique is based on Michelson's interfometer and has been widely described., ${ }^{1,2}$ For ophthalmic systems, a low-coherence $820 \mathrm{~nm}$ wavelength pulsed source is used. Shorter wavelengths allow improved axial resolution, but there is a trade-off with reduced tissue penetration and sensitivity. Echoes from the eye tissue are combined with a variable delay reference pulse. Correlation (a mathematical description for, in essence, what means a type of combination of the two signals) between these two signals provides data on fluctuations of scattered light intensity as a function of time. Plots of this A-line (by analogy to ultrasound imaging) are as either brightnessmodulated spots, the brightness of the spots indicating the amplitude of the echo or more commonly, a false colour is ascribed to particular amplitudes (Figure 1). The convention is to show high-amplitude echoes in warm shades (red, yellow, and white) and lowamplitude echoes in cold shades (blue, green, and black). The position of the spot indicates the depth in tissue from which the echo was generated. A large series of such A-lines are generated to produce the OCT image.

\section{Fourier-domain OCT}

Recent advances in component specifications and reliability have allowed the practical implementation of fourier-domain OCT. ${ }^{3}$
Department of Ultrasound, Moorfields Eye Hospital, London, UK

Correspondence: M Restori, Ultrasound, Moorfields Eye Hospital, London EC1V 2PD, UK.

Te1: + 020756622 17;

Fax: +020756 62217

E-mail: restoriharrison@ blueyonder.co.uk

Received: 18 January 2008 Accepted in revised form: 18 January 2008 Published online: 22 February 2008 


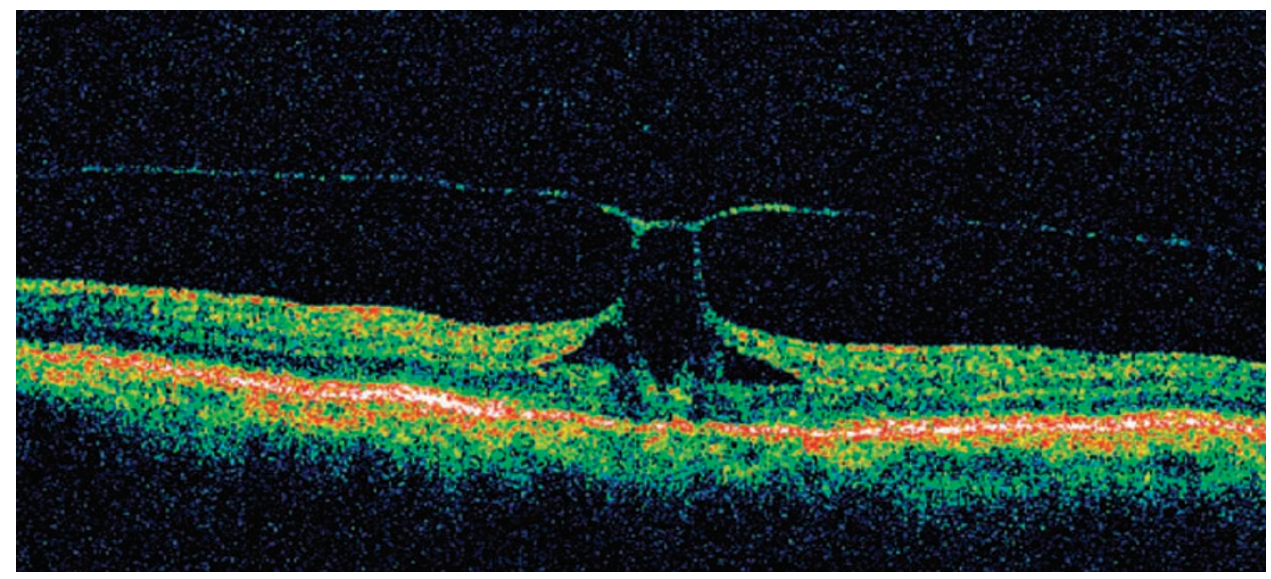

Figure 1 Time-domain OCT image: PVD; pseudo macular hole (Status OCT 3 (Carl Zeiss Meditec Inc.).

a

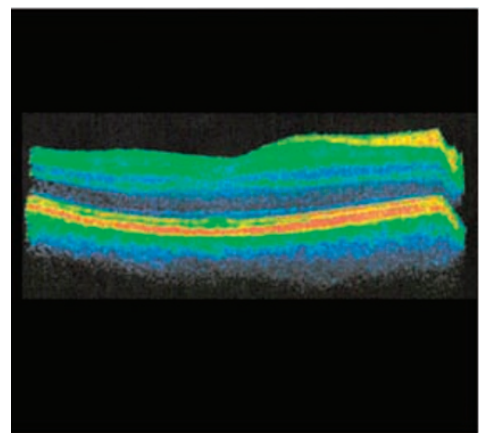

C

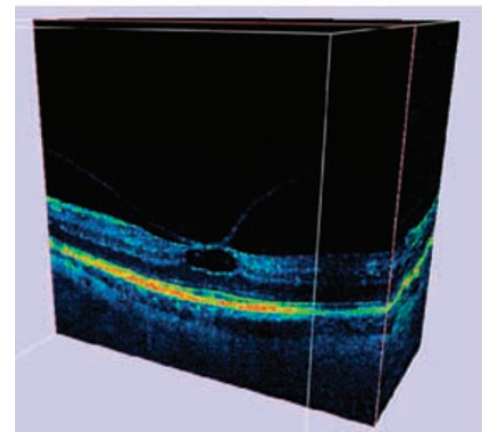

b

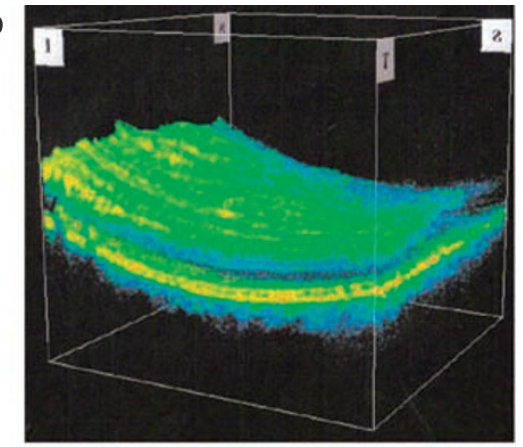

d

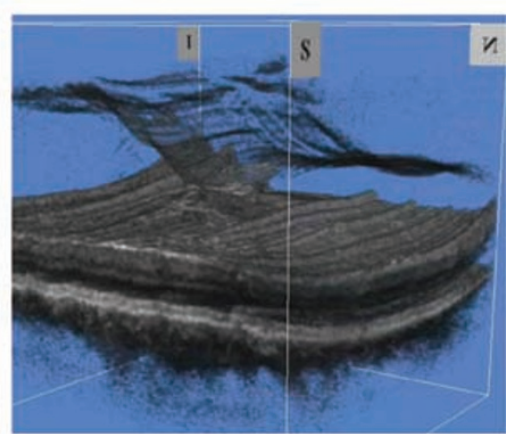

Figure 2 Fourier-domain OCT images: (a and b) Retinal layers (Topcon 3D 1000). (c) PVD;cyst (S OCT Copernicus). (d) PVD shown in 3D inserting into macular hole (Topcon 3D 1000).

A broad-spectrum light source (alternatively, the frequency of a narrowband continuous wave can be swept. This obviates the need for a spectrometer) is used to illuminate the sample and the depth information is extracted from the interference spectrum of the scatteredtissue signal. The scattered-light intensity fluctuations are combined with a broadband reference beam as same as the interrogating beam and are then passed through a spectrum analyser and the output sent to an array of detectors. The resultant output interference power spectrum undergoes mathematical manipulation (fourier transform) to produce the correlation function from which the depth information is read.

This technique has the advantage that it allows improved axial resolution. The technique is considerable faster than the time-domain OCT and allows more A-scan lines producing improved lateral resolution, signal to noise and sensitivity. Faster time allows the use of 3D imaging software at practical speeds (Figure 2) allowing data to be displayed as individual B-scans, 
animated 3D data cubes, or serial sections within a data cube, which can be viewed in 'fly-through' timed sequence.

\section{Ultrasound imaging}

Diagnostic ultrasound is used in the assessment of eyes in which opaque ocular media precludes ophthalmoscopic visualisation of the fundus.

Real-time B-mode imaging and colour flow mapping (CFM) are used in the diagnosis of vitreoretinal disease.

\section{Real-time B-mode imaging and CFM}

Patients are examined seated with the head positioned and stabilised with a chin rest and forehead support. The probe is smeared with a coupling gel, applied to the closed eyelid and moved in a systematic fashion over the eyelid to produce a series of B-mode sections. Each probe position corresponds to a B-mode section. Images are refreshed at rates of up to $25 \mathrm{~B}$-scans/s. Dynamic studies are performed by asking the patient to deviate the eye while the probe is held in a stationary position.

Real-time B-mode imaging illustrates the dynamic and topographical characteristics of pathology. CFM is used to image blood flow. The absence of blood CFM can be used to distinguish the avascular vitreous from vascular tissues. All images shown were taken with a Sequoia 512 (Acuson: Siemens).

\section{The vitreous}

The normal vitreous cavity on ultrasound B-mode section (Figure 3a) appears echolucent. Typical axial resolutions at $12 \mathrm{MHz}$ are $130 \mu \mathrm{m}$ and lateral resolutions $800 \mu \mathrm{m}$. Optical opacities may give rise to echoes of varying amplitudes. Generally, inflammatory debris cannot be distinguished from haemorrhage (Figures 3b,
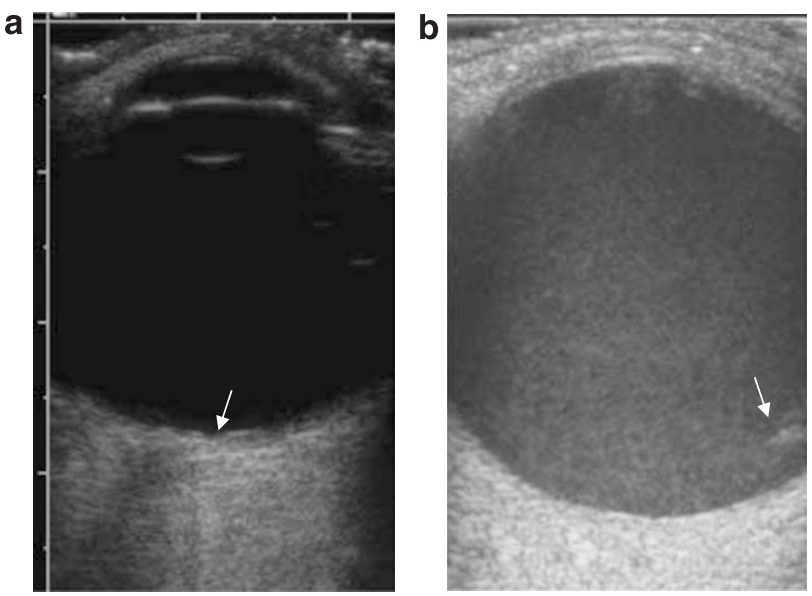

e
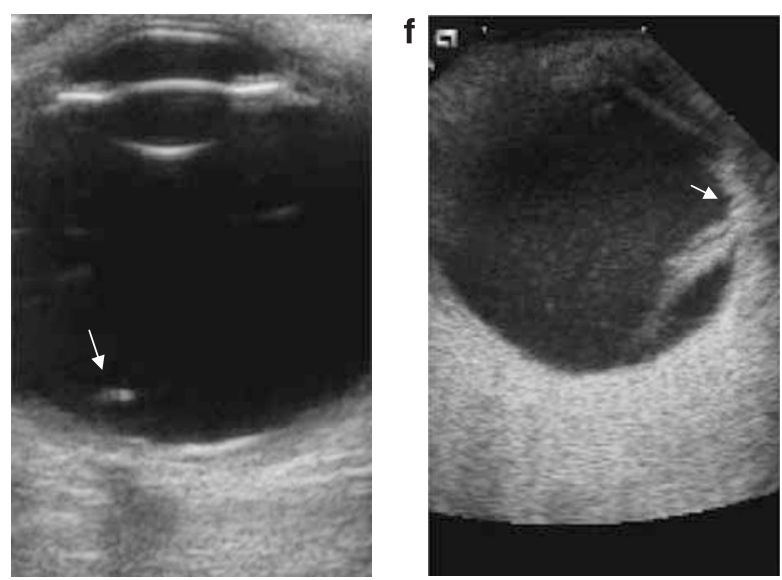
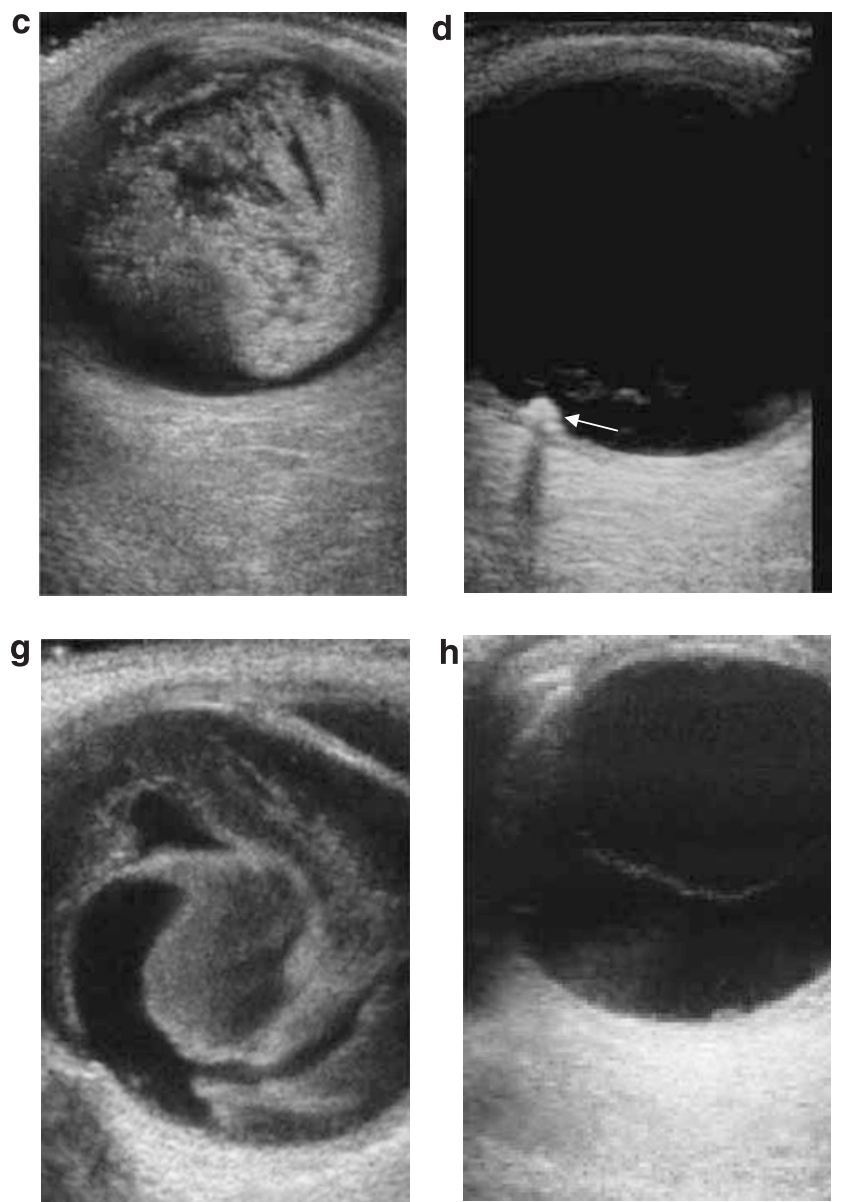

Figure 3 Transverse ultrasound B-scans: (a) Central section: normal vitreous; foveal dip (arrow). (b) Post-vitrectomy haemorrhage; residual gel frill (arrow). (c) Intragel asteroid hyalosis; PVD. (d) Intraocular foreign body on retinal surface (arrow) casting shadow; PVD. (e) Intravitreal larva (arrow). (f) Intravitreal bleed with source (arrow) indicated by region of fresh highly echogenic haemorrhage. (g) Intravitreal haemorrhage with lacunae; no PVD. (h) Optically clear vitreous; PVD. 
a

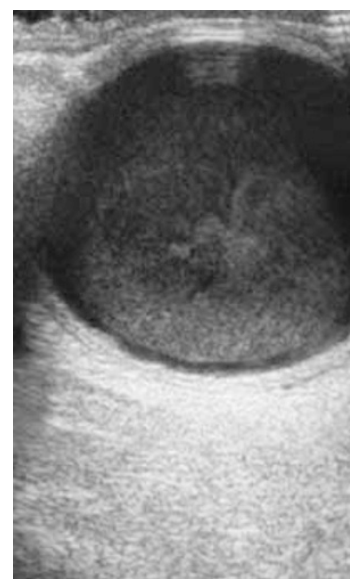

e

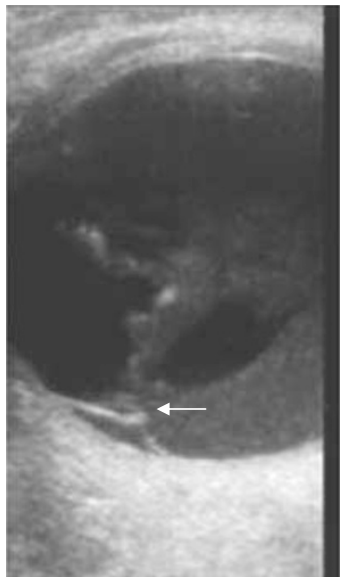

b

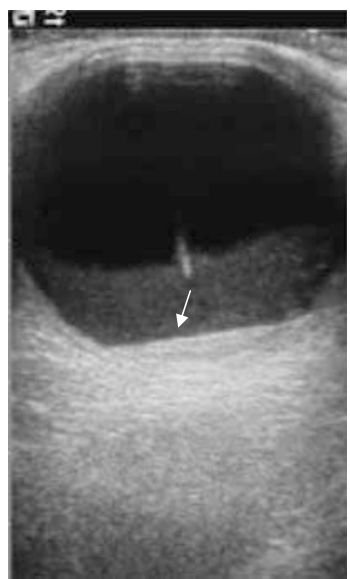

f

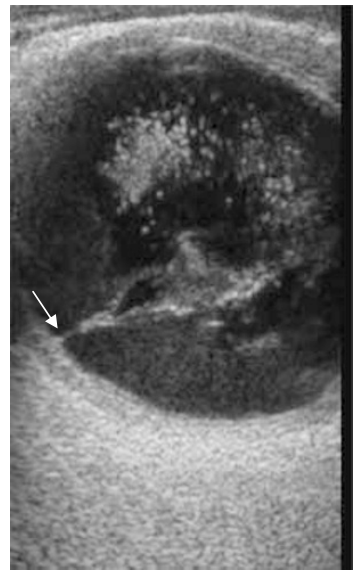

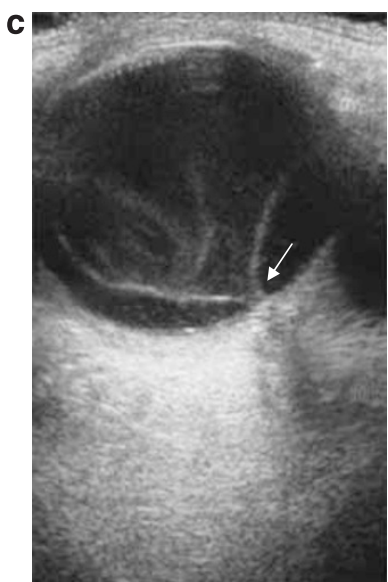

d

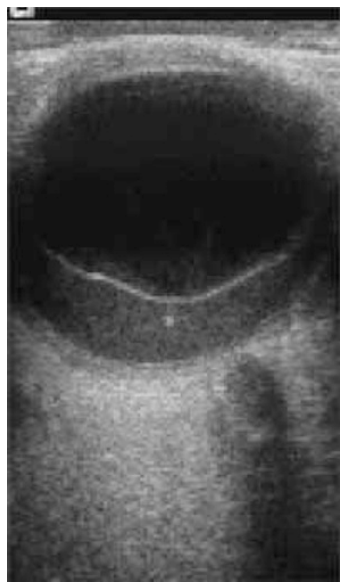

g

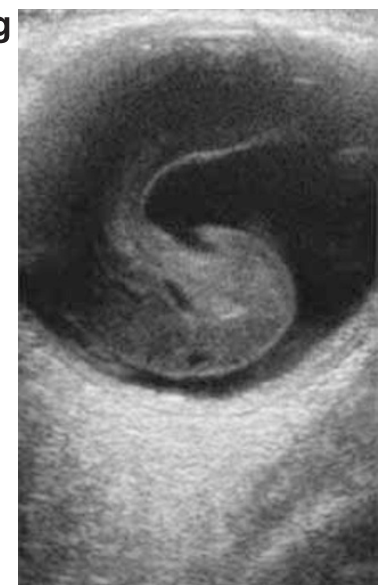

h

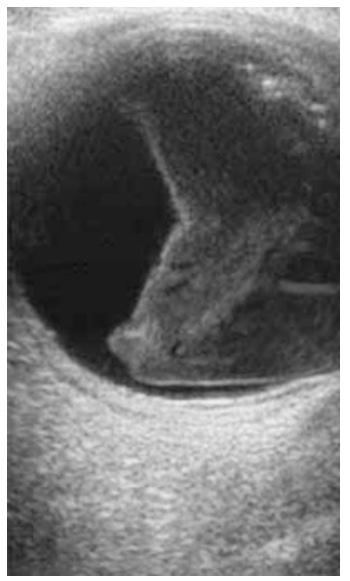

Figure 4 Transverse ultrasound B-scans. (a) Intragel haemorrhage outlining PVD; fluid level (arrow). (b) Retrohyaloid haemorrhage outlining PVD; fluid level (arrow). (c) Intragel and retrohyaloid haemorrhage; incomplete PVD (disc adhesion; arrow) outlined by fresh haemorrhage on posterior hyaloid interface. (d) Intragel and retrohyaloid haemorrhage; PVD outlined by fresh haemorrhage on posterior hyaloid interface. (e) Deviated gaze; central gel and retrohyaloid haemorrhage; clear cortical gel; temporal retinal tear (arrow). (f) Deviated gaze; intragel asteroid hyalosis; retrohyaloid haemorrhage; vitreoretinal adhesion at site of branch vein occlusion (arrow). (g) Laterally deviated gaze to demonstrate gel movement: intragel haemorrhage. (h) Same eye as (g) Nasally deviated gaze to demonstrate gel movement: intragel haemorrhage.

$\mathrm{d}, \mathrm{f}-\mathrm{h}, 4$, and 5). Calcium-laden asteroid hyalosis (Figures $3 \mathrm{c}$ and $4 \mathrm{f})$, however, is clearly distinguishable from other opacities as it generates very high-amplitude echoes. Foreign bodies give rise to very high-amplitude individual echoes and may cast a shadow (Figure 4d), but may show comet tail type (Figure 5a) reverberation artefacts. Small gas bubbles in the vitreous can be distinguished from foreign bodies by head positioning.

The presence of lacunae within dispersed vitreous opacities suggests probable progression to posterior vitreous detachment (PVD). In such instances, care must be taken not to confuse posterior located lacunae (Figure $3 \mathrm{~g}$ ) with the posterior hyaloid face.

\section{Posterior vitreous detachment}

PVD in an eye with an optically transparent vitreous is seen on the B-scan as a faint line (Figure 3h), which moves sinuously during eye deviations. The echoes from the interface of gel and retrohyaloid fluid are very low in amplitude due to the similarity of acoustic impedance (density $\mathrm{X}$ velocity of sound in medium) between the gel and clear retrohyaloid fluid.

The presence of vitreous opacities aids in the ultrasound diagnosis of vitreous detachment allowing the outline of the posterior hyaloid interface to be clearly delineated. Various patterns of PVD exist. Echoes arising from fresh haemorrhage (Figure $4 \mathrm{c}$ and d) or fibrous tissue scattered from the detached gel boundary (Figure 5h) may outline the PVD. More commonly, diagnosis of PVD can be made when echoes arising from opacities are compartmentalised to and fill either the vitreous gel (Figures $3 c$ and $4 a$ ) or the retrohyaloid space (Figure 4b). Retrohyaloid haemorrhage frequently bleeds into the central gel 

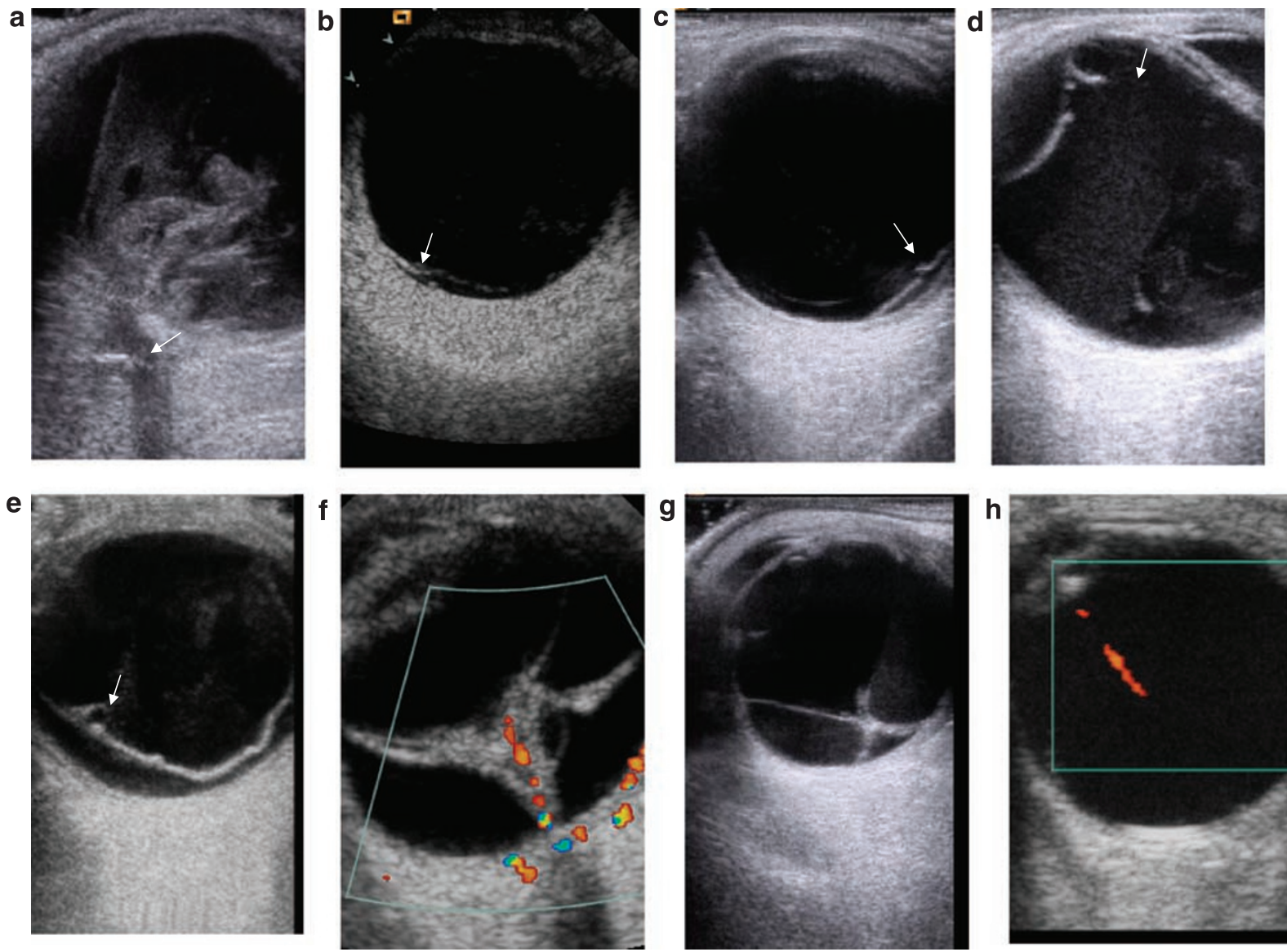

Figure 5 Ultrasound B-scans and CFM. (a) Deviated gaze; PVD incarcerated by a wide adhesion into the posterior temporal retina; intragel haemorrhage; posterior scleral rupture; intraconal foreign body adjacent to nerve (arrow) with comet-tail reverberations. (b and c) PVD inserting into anterior edge of retinal tear (arrow); intragel haemorrhage. (d) Deviated gaze; PVD inserting into anterior edge of retinal tear (arrow); localised temporal retinal detachment; central gel and retrohyaloid haemorrhage. (e) PVD inserting into retinal tear (arrow); intragel haemorrhage; rhegmatogenous retinal detachment. (f) CFM: PVD; fixed retinal detachment showing blood flow in red. (g) PVD with partly fibrotic posterior hyaloid membrane inserted by fibrous stalk into tractional retinal detachment; retrohyaloid haemorrhage. (h) CFM: blood flow (red) in patent persistent hyaloid vessel.

leaving clear cortical gel (Figures $4 \mathrm{e}$ and $5 \mathrm{~d}$ ). All patterns exhibit a common feature on dynamic B-mode study in that the internal gel echoes move as a corporate entity (Figure $4 \mathrm{~g}$ and $\mathrm{h}$ ) flopping from side-to-side on deviations of gaze.

Often a development adhesion of the detached gel to the disc (Figure 4c) exists. Other vitreoretinal adhesions may indicate the sites of new vessels or a branch vein occlusion (Figure 4f). In trauma, the detached gel may be incarcerated into other tissues as suggested by adhesions (Figure 5a) and an asymmetrical suspension of gel on B-scan section. Vitreoretinal adhesions may be tenuous (Figure $4 \mathrm{f}$ ) or more extensive (Figure 5a) and are best illustrated using dynamic studies, which aid in the appreciation of tractional forces on the retina around the base of such adhesions.

\section{Post vitrectomy and silicone oil}

Post-vitrectomy haemorrhage (Figure $3 b$ ) gives rise to a uniform distribution of haemorrhage and the residual gel frill is detectable.

The presence of silicone oil (Figure 6) makes images more difficult to interpret. Artefacts are caused by the differences in the oil properties compared with biological tissue. The speed of sound in silicone oil, only two-thirds of that in biological tissue, is not corrected for by the scanner and causes the vitreal length to appear elongated on the display by a factor of 1.5 .

\section{Retinal tears and retinal detachment}

Retinal tears (Figures $4 \mathrm{e}, 5 \mathrm{~b}$ and $\mathrm{c}$ ) are imaged as two strong echo tags. During eye movements, the gel can be 

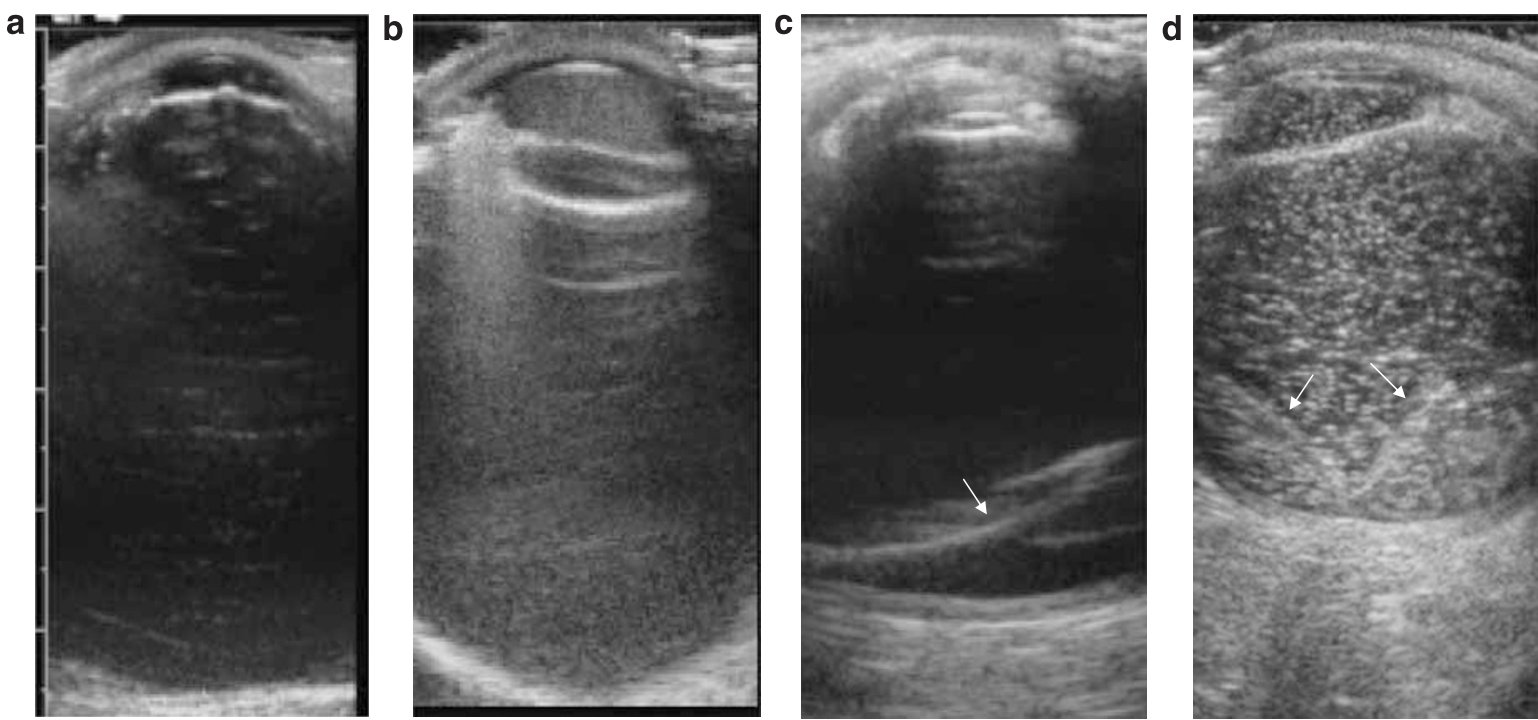

Figure 6 Ultrasound B-scans : silicone oil. (a) Aphakia; silicone oil-filled vitreous; low-sound speed in oil elongates the appearance of vitreous. (b) Emulsified silicone oil in anterior chamber and silicone oil and haemorrhage in vitreous. (c) Longitudinal section; silicone oil in vitreous; inferior half retinal detachment (arrow). (d) Post-silicone oil removal; residual oil droplets in anterior chamber and vitreous; total retinal detachment (arrows) with subretinal silicone oil droplets.

imaged inserting into the more anterior echo tag. A drop in the retinal level should be discernible posterior to the two echo tags.

Differentiation between incomplete PVD tethering to the disc and total detached retina is based on several features. The echogenicity of the detached retina is usually higher than that from the posterior hyaloid interface, although the presence of fresh haemorrhage or fibrous tissue along the posterior hyaloid interface may give equally high-amplitude echoes. Importantly, in PVD, only one interface can be imaged even at high settings of gain during dynamic scanning. For diagnosis of retinal detachment (Figure $5 \mathrm{~d}-\mathrm{g}$ ) in adults, two interfaces should be imaged, one from the incomplete PVD and the other from the detached retina. The vitreous flops from side-to-side during deviations of gaze (Figure $4 \mathrm{~g}$ and $\mathrm{h}$ ), whereas fresh rhegmatogenous retinal detachment demonstrates an undulating type of motion, which continues for a short period after the eye has become stationary. In rhaegmatogenous retinal detachment, the anterior edge of the retinal tear can be localised by delineating the site of vitreoretinal adhesion (Figure 5e and f) during dynamic scanning.

The absence of blood vessels in the vitreous (except in patent primary hyperplastic primary vitreous; Figure $5 \mathrm{~h}$ ) can be used as further differentiation criteria. Blood flow can be detected even in longstanding retinal detachment (Figure $5 \mathrm{~g}$ ), using CFM techniques.

Caution should be used in exclusion of retinal detachment in the absence of detected blood flow, as this may indicate the lack of sensitivity of the CFM modality or inexperience of the operator.

\section{Conclusions}

OCT offers in vivo, non-contact, static images of the vitreous and posterior coats, to the depths of typically $2 \mathrm{~mm}$ with both unprecedented resolution (micron scale) and speeds, in optically transparent and translucent tissue.

Ultrasound imaging offers in vivo, direct contact, dynamic images of the entire globe, and orbit, typically to depth of $50 \mathrm{~mm}$ with lower resolutions ( $100 \mu \mathrm{m}$ scale) in optically transparent, translucent, or optically opaque media.

\section{Acknowledgements}

All OCT images are courtesy of Medical Illustration Department, Moorfields Eye Hospital, London. I thank Drs Sidney Leeman and Andrew Healey for their helpful comments.

\section{References}

1 Huang D, Swanson EA, Lin CP, Schuman JS, Stinson WG, Chang W et al. Optical coherence tomography. Science 1991; 254(5035): 1178-1181.

2 Fujimoto JG. Optical coherence tomography for ultrahigh resolution in vivo imaging. Nat Biotechnol 2003; 21(11): 1361-1367.

3 Huber R, Taira K, Wojtkowski M, Ko TH, Fujimoto JG. Highspeed frequency swept light source for fourier domain OCT at $20 \mathrm{KHz}$ a-scan rates: photonics West-Bios 2005. Coherence domain optical methods and optical coherence tomography in biomedicine $1 \times$ (B0114). 